\title{
COLLAPSE OF A CAVITATION BUBBLE GENERATED BY LOW VOLTAGE DISCHARGE IN WATER
}

\author{
Miloš Müller, Jiří Unger, Tomáš Bud'arek, Patrik Zima• \\ Abstract: The article presents experimental results of the optical study of \\ cavitation bubble collapse close to a solid boundary in water. The bubble was \\ generated by discharge of two low-voltage capacitors. High-speed CCD camera \\ was used to record the time evolution of the bubble size. High-power halogen lamp \\ was used for illumination. The system was synchronized by pulse generator \\ connected to an oscilloscope. The velocity of the re-entrant jet was estimated from \\ the time resolved photography for different maximum bubble sizes.
}

\section{INTRODUCTION}

Spark-generated cavitation bubbles represent one of the possibilities available for the investigation of single bubble behaviour. This method is usually used to investigate large bubbles [1] since the maximum bubble radius is required to be greater than the diameter of the electrodes. The electrodes are usually placed far from each other and very high voltage is necessary for bubble creation. We used a low voltage technique described in [2], which produces bubbles similar to those produced by a laser.

According to theory the bubble collapsing close to a solid wall produces a rapid re-entrant liquid microjet. This microjet is understood to be responsible for the material damage of the solid surface [3]. The average impulsive force of the jet can be calculated from its velocity and its diameter.

\section{EXPERIMENTAL SETUP AND MEASUREMENT}

Figure 1 shows the experimental setup for investigation of spark-generated cavitation bubbles. The bubble is created inside the water bath. The electrodes are position very close to each other to enable the use of low voltage. The spark is produced by two parallelly connected capacitors $(60 \mathrm{~V}, 2200 \mu \mathrm{F})$, which are discharged through a relay. The relay is controlled by a hand trigger, which is also connected to the signal generator. After some time given by the time response of the relay recording by the CCD camera is initialized. The illumination is provided by continual white light produced

\footnotetext{
- Miloš Muller, Faculty of Mechanical Engineering, Technical University of Liberec, Hálkova 6, 46117, Liberec 1, Czech Republic, milos.muller@tul.cz

Jiri Unger, Faculty of Mechanical Engineering, Technical University of Liberec, Hálkova 6, 46117, Liberec 1, Czech Republic, jiri.unger@tul.cz

Tomáš Bud'árek, Faculty of Mechanical Engineering, Technical University of Liberec, Hálkova 6, 46117, Liberec 1, Czech Republic, T.Budarek@seznam.cz

Patrik Zima, Department of Thermodynamics, Institute of Thermomechanics, v. v. i., Academy of Sciences, Dolejškova 5, 18200 Praha, Czech Republic, zimap@it.cas.cz
}

This is an Open Access article distributed under the terms of the Creative Commons Attribution License 2.0, which permits unrestricted use, distribution, and reproduction in any medium, provided the original work is properly cited. 
by the Fomei DIGITAL 1200 halogen lamp. The illumination was homogenized using dull finish glass. The diameter of the electrodes at the discharge position was $0.2 \mathrm{~mm}$. The electrodes had to be treated after each discharge. Three measurements were taken, the impact loads were determined for two of them.

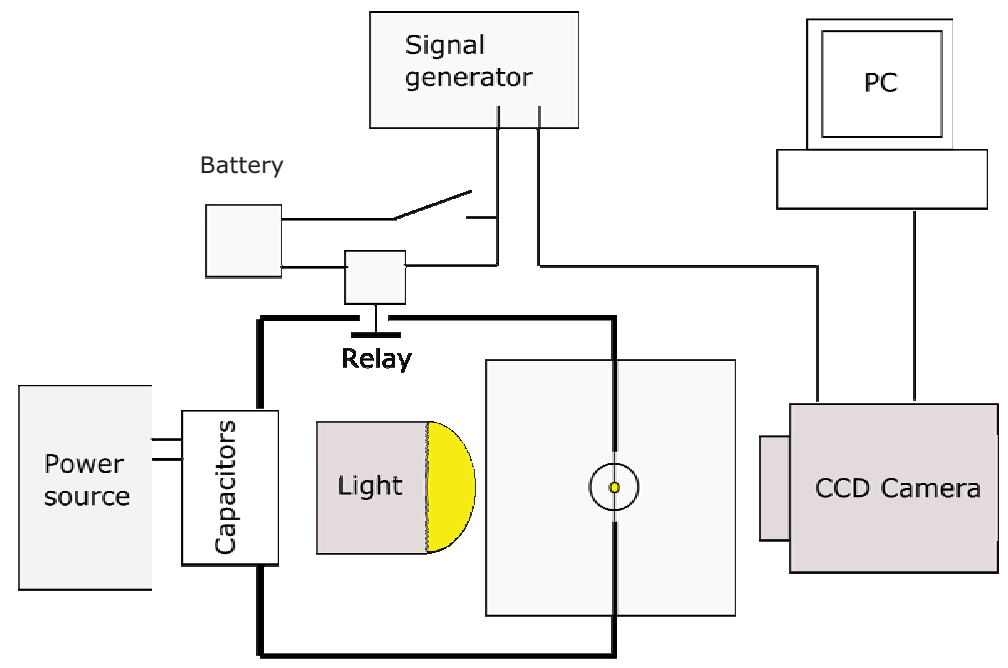

Figure 1. Experimental setup

\section{REsults}

Figure 2 shows a sequence of images of a typical spark-generated bubble collapse. To evaluate the time history of bubble radii in vertical and horizontal direction we introduced $R_{h} / R_{0}$ a $R_{h} / R_{0} . R_{h}$ and $R_{v}$ are horizontal and vertical bubble radii and $R_{0}$ is the electrode diameter. At the beginning, the spark generates plasma, which then evaporates the liquid around it. The violent plasma expansion initiates the liquid movement and produces several other side effects described in [4]. Then, the velocity of bubble expansion is decreased and the bubble reaches its maximum radius. Parts of the electrodes are visible inside the bubble, however, the size of electrodes is much smaller than the bubble radius. The bubble expansion is followed by compression and collapse. During the first rebound re-entrant jet is formed and directed towards the wall. The deformation of the bubble is evident from Figures 3 and 4 . The force exerted by the bubble jet on the surface can be calculated from the following equation

$$
F=\rho S v v=\rho \dot{V} v
$$

where $S$ is the bubble jet surface, $v$ is the jet velocity and $\rho$ is the liquid density. The results of the calculation are summarized in Table 1.

\begin{tabular}{c|c|c|c|}
\hline Maximum bubble size & Jet velocity & Jet diameter & Force load \\
\hline$[\mathrm{m}]$ & {$[\mathrm{m} / \mathrm{s}]$} & {$[\mathrm{m}]$} & {$[\mathrm{N}]$} \\
\hline 0,0032 & 15,5 & 0,0015 & 0,42 \\
\hline 0,0034 & 17,5 & 0,0014 & 0,47 \\
\hline 0,0046 & $\mathrm{x}$ & $\mathrm{x}$ & $\mathrm{x}$ \\
\hline
\end{tabular}

Table 1. Impact load (force) generated by the bubble jet 


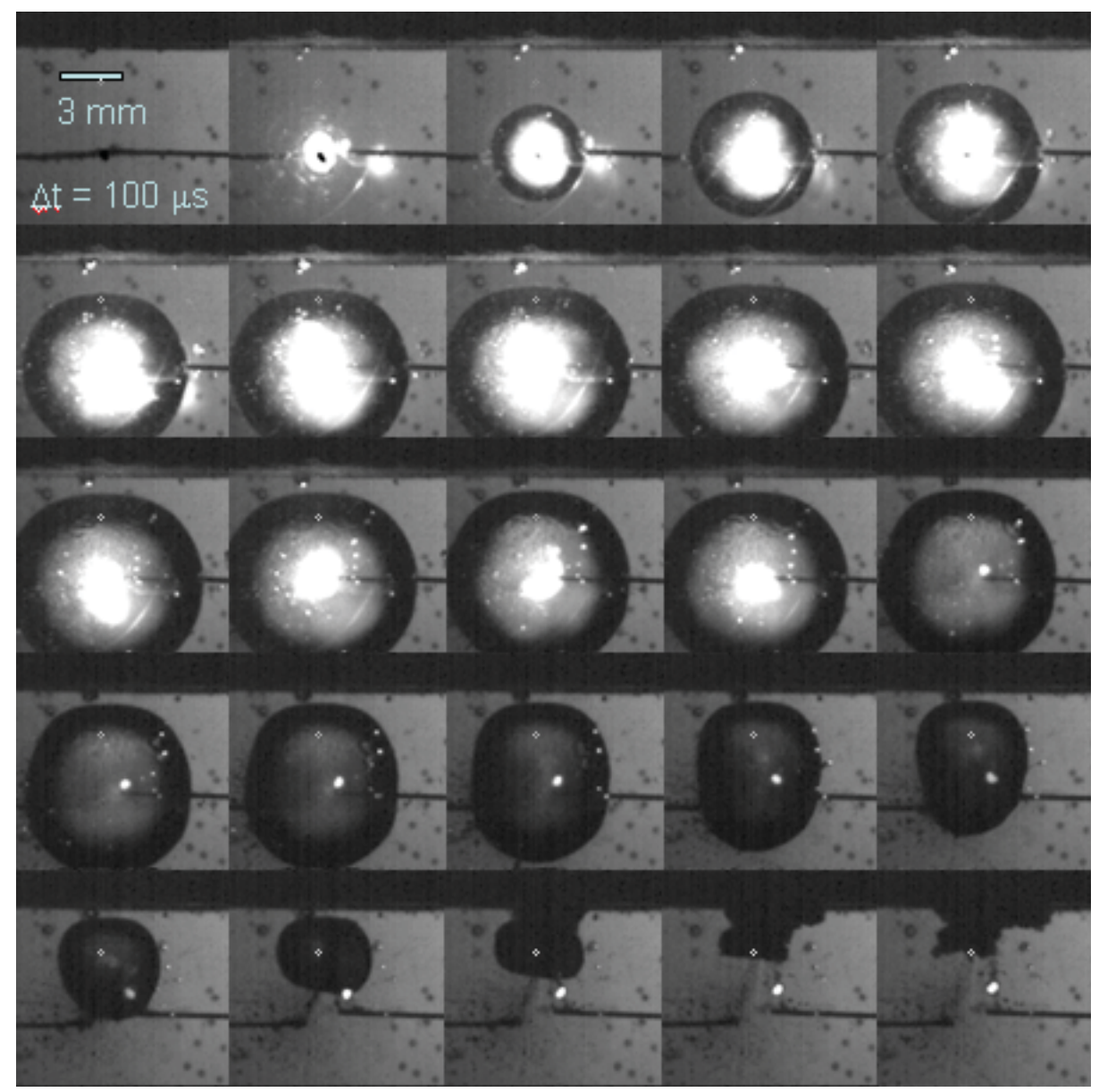

Figure 2. Bubble collapse

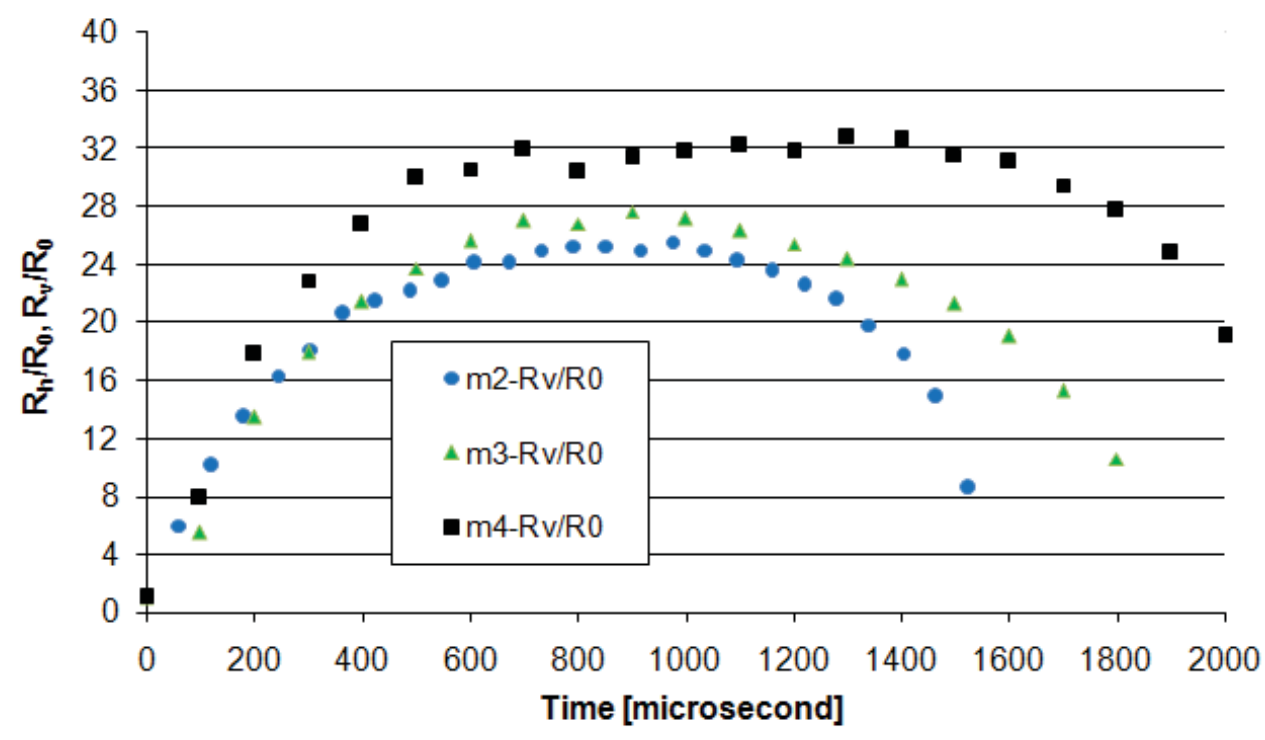

Figure 3. Time evolution of the vertical bubble radius 


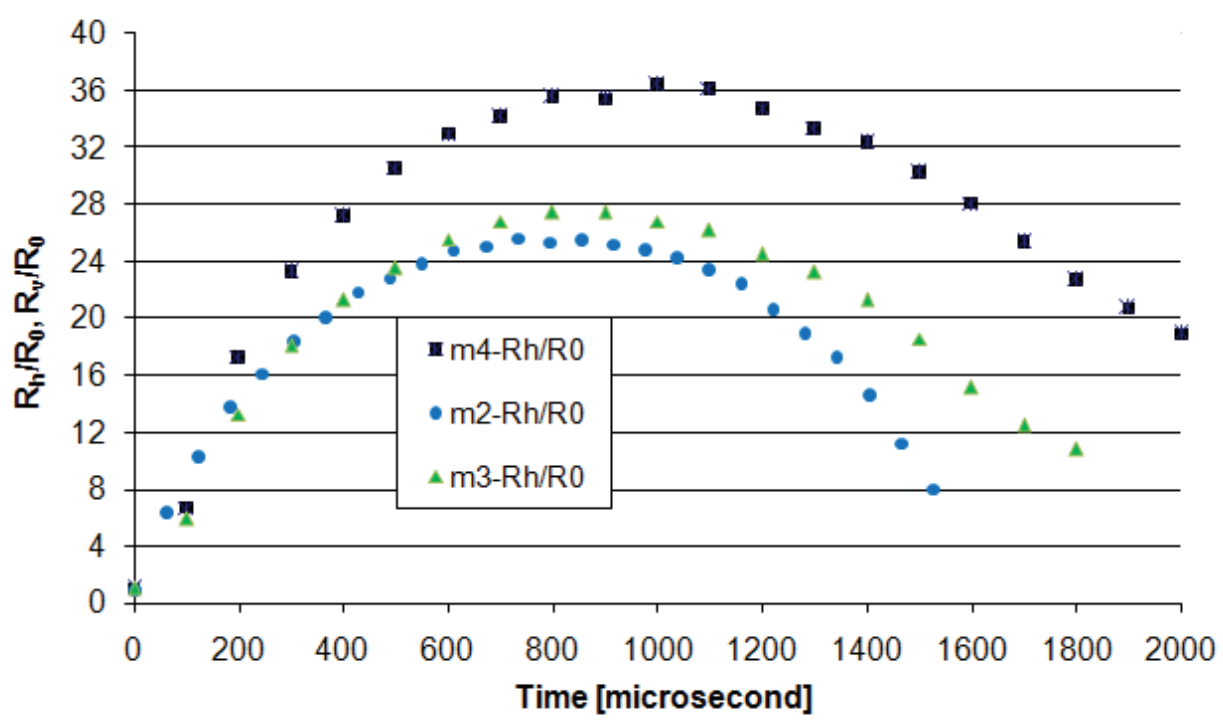

Figure 4. Time evolution of the horizontal bubble radius

\section{Conclusion}

Collapses of spark-generated cavitation bubbles were investigated. The values of the impact forces were evaluated for two measurements. The preliminary results show strong deformation of the bubble radius during the first compression and the first rebound. In addition, one can observe melting of the electrodes during the plasma creation.

\section{ACKNOWLEDgement}

The authors wish to thank the Czech Science Foundation for providing financial support for grant no. 101/10/1428 and grant no. MSM 4674788501 of the Technical University of Liberec.

\section{REFERENCES}

[1] Chahine G. L.: Spark Generated Bubbles as Laboratory-Scale Models of Underwater Explosions and their use for Validation of Simulation Tools, Proceedings of the 66th Shock and Vibration Symposium,1995, Biloxi, MS

[2] Turangan C.K., Ong G.P., Klaseboer E., Khoo B.C.: Experimental and numerical study of transient bubble-elastic membrane interaction, Journal of Applied Physics, Volume 100, Issue 5, 2006, pp. 054910-054910-7

[3] Brennen Ch. E.: Cavitation and Bubble Dynamics, Oxford University Press, 1995

[4] Noack J., and Vogel A.: Laser-Induced Plasma Formation in Water at Nanosecond to Femtosecond Time Scales: Calculation of Thresholds, Absorption Coefficients, and Energy Density, IEEE J. Quantum Electron., 35, 1999, pp. 1156-1167 